\title{
THE COMMUNICATION AUDIT: INFORMATION FLOW AND THE ORGANIZATION
}

\author{
MAUREEN S. BOGDANOWICZ \\ CALGARY, ALBERTA
}

\begin{abstract}
The Communication Audit is a process of exploring, examining, monitoring, and evaluating the communication process in organizations. In organizational settings, discourse serves either to maintain established policies, procedures, and operations or to facilitate change. Members of organizations, at all levels, have preferences for when, from whom, and in what media they receive information and provide feedback. Rhetorical theory and discourse analysis include audience and setting in a review of the process of communication. Recently, researchers in organizational behaviour have proposed formal reviews of the communication process, particularly from the perspective of staff who receive messages. Often preferences vary. In different instances, different forms and sources of communication are the most salient, informative, valued, and preferred.
\end{abstract}

\section{INTRODUCTION}

As we approach the twenty-first century, we will likely witness the reversal of the old saw, "The more things change, the more they remain the same." Now, change is the norm, and coping with change and its accompanying stress is fertile ground for consulting, advising, and counselling. If we follow the advice of the gurus, we should powershift into megatrends and learn to dance with giants and swim with sharks (Naisbitt and Aburdene, 1990; Toffler, 1990; Kanter, 1989, MacKay, 1988). Increasingly, organizations are being asked to do more with less, work smarter not harder, tighten belts, cut fat. In such an environment, flexibility is not only rewarded, but required. 
Academic institutions, too, are forced to cope with accelerating change. Lines between disciplines are disappearing as team approaches to interdisciplinary teaching and learning succeed. Those of us who teach communication, in all its forms, have long been involved with "writing across the curriculum," for instance. We recognize the integral role of good communication skills for our engineers, scientists, accountants - for all academic disciplines. Increasingly, we involve "workplace" consultants as guest speakers, team markers, research associates, and, perhaps most importantly, as future employers of our graduates. When they enter the workforce, our graduates must communicate within the corporate cultures of the industries and organizations they join. Thus, it is contingent on us to be familiar with the discourse of the workplace and to incorporate this discourse in our classrooms.

The environment of discourse in an organization includes general staff meetings where the President or CEO makes announcements; formal reports and proposals to decision makers, like Boards; informal reports and proposals within departments; memos in many levels of formality; staff meetings; one-on-one discussions and interviews; the company newsletter; department bulletins; bulletin boards; coffee shop chatter; and, perhaps most effective, the company grapevine. What mode and source of communication is most effective, and when?

Communication Audits have been introduced in some organizations to evaluate communication flow and determine how best to communicate within specific corporate cultures. Findings are often illuminating: staff preferences for modes and sources of information vary. For instance, Communication Audits have found that, generally, staff prefer to hear from their supervisor of changes that affect their day-to-day work. Moreover, some want and need a memo to maintain in their cavernous files. For them, if an announcement is not in writing, it is not valid. Managers are often surprised to find, through a Communication Audit, that their carefully crafted written discourse to all staff is frequently ignored and that their staff claim to have heard of corporate decisions either in the company newsletter or over a coffee. Frequently, information is distorted in the communication chain, and the staff is not well informed of reasons for executive decisions, despite their having attended a general meeting or having received a written announcement. 
If our function as teachers of technical communication is to equip our graduates to communicate in the workplace, we need to include the issues of communication flow in our courses. It is not enough to teach The Memo, The Letter, The Formal Report, The Feasibility Study, The Oral Presentation, and every other topic in the communication course. We need to address the appropriateness of each mode individually and as it is combined with others in the real world of the workplace. Only then will we have included the full environment of corporate discourse in our courses. Only then will our engineers, scientists, accountants, and graduates in general be prepared to communicate their findings on the job.

\section{CHANGE AS THE NORM IN THE WORKPLACE}

Organizations increasingly meet challenges to complete in the global workplace by implementing changes, particularly changes involving automation of information. Such changes involve alterations in the nature of work and the environment of the workplace. The impact of automation on workers has been the subject of insightful analysis, notably in the recent work of Shoshana Zuboff at Harvard and Heather Menzies of Carleton. Both cite the communication process as a critical issue in how change is implemented by management and accepted by workers. Management is challenged to keep staff informed about, involved with, and committed to any functional changes it plans.

Communication is critical. Increasingly, organizations are becoming aware that organizational change and development is in actuality a human resource issue. Since change affects staff in significant ways, the staff must feel a sense of ownership of the directions in which their organization is moving. They must be informed of the need for change and be engaged in two-way communication about proposed changes and ways of implementing them. "[In] a recent IBM survey...questions asked 785 opinion leaders in the human resources field what they thought were the most effective elements of human resources policy. 'Open communication' topped the list" (Hopkins, Nestleroth, and Bolick, 1991, p. 202). Such "open communication" is especially critical when change is an issue. 
Change will continue to be an issue. The 1990's demand continual adoption, adaption, and ongoing learning and integration. Research in Organizational Development reviews the necessity to involve affected staff early and continually in change. (See especially Leonard-Barton, 1985, 1988 and Hopelain, 1982). Effective communication can reduce the stress associated with change.

Richard Paul Wurman (1989) has identified a typical dysfunction in this data rich, but information poor, decade: Information Anxiety. This stressful condition "is produced by the ever-widening gap between what we understand and what we think we should understand. It is the black hole between data and knowledge and it happens when information doesn't tell us what we need or want to know." He explains, "We learn through context, through what surrounds, informs, and opposes an idea" (p. 72). The implications are clear for those of us who teach technical and organizational communication. We are adept at linguistic and rhetorical analyses of units of discourse: a memo, report, article, letter. However, communicators must contextualize each unit of discourse, particularly when such discourse is intended to smooth organizational functioning.

\section{THE COMMUNICATION AUDIT}

The Communication Audit is a process of exploring, examining, monitoring, and evaluating the communication process in organizations. Ideally, such an audit can be informative, descriptive of efficient and effective communication flow; often, however, it is remedial, commissioned to identify flaws in the communication flow.

What can an organization learn from a Communication Audit? An audit can identify agreement or significant differences among answers to these questions:

1. How does management perceive the information flow in the organization? How should it flow ideally? How does it flow in actuality? Do management's perceptions of the ideal and actuality mesh? 
2. How does the staff perceive the information flow in the organization? How does it actually flow?

3. How would staff prefer information to flow? What is the ideal flow from a staff perspective?

Generally, the Communication Audit is enlightening because it identifies snags and gaps in a process, not in individuals. Communication difficulties are depersonalized and, thus, not personally threatening to resolve.

Data in a Communication Audit are collected in a number of ways, depending on the size, culture, formality, and complexity of the organization, division, or work unit under scrutiny. Typical techniques include observations, interviews, questionnaires, critical incidents, network analysis, content analysis, and communication diaries (Downs, 1988, p. 16). Generally, combinations of two, three, or four of these seven techniques comprise the audit. They are discussed here individually.

\section{Observations}

An auditor sits in on structured meetings, interviews, and discussions as well as unstructured situations, including social situations like coffee breaks and lunches. She observes the discourse flow and general communication climate. Of course, such observation is seldom unobtrusive and data collected are generally soft. A positive product of observations, however, is a sense of the organizational attitude toward discourse: modes of address, levels of formality, and diversity of participants and participation.

2. Interviews (and Focus Groups)

In-depth interviews with randomly selected individuals representing a cross section of the organization often result in valuable data. Of course, such exercises are costly in terms of time, both the auditor's and the subjects'. Focus groups, group discussions focussed on an issue, are more efficient in terms of time than individual interviews. However, unless 
the auditor is skilled in conducting group sessions, not all participants will engage fully and candidly. One-on-one interviews allow for clarification of terminology and expanded responses to open-ended questions. If she capitalizes on the personal contact of the interview or focus group, the auditor can gather particularly valuable nonverbal data as well as data that differentiate between staff preferences and actuality in the organizational communication flow.

\section{Questionnaires}

The questionnaire is a time-efficient auditing tool. However, it is crucial that the instrument be well constructed and tailored to the particular organization under study. Often, focus groups and interviews precede the auditor's constructing a questionnaire and follow her administering it. The earlier personal contacts identify issues to include in the questionnaire; the follow-up provides clarification of issues that emerge unexpectedly. Unlike the interview and focus group, the questionnaire can be targeted to all members of the organization. If well constructed, it need not be excessively costly in terms of time required to complete it. A well constructed questionnaire asks appropriate questions, and, if it is multiple choice, does not provide leading responses. If the auditor is not trained in and proficient at developing questionnaires, this instrument may provide data which distort the facts.

\section{Critical Incidents}

The basic objective of the critical incident method is "to focus on concrete behaviours while eliminating statements of opinion, gross generalizations, imprecise evaluations, and stereotypes" (Downs, 1988, p. 133). A communication auditor can include "critical incident" items in questionnaires, interviews, or focus groups. The respondent is asked to think of a particularly effective and/or ineffective communication 
event and answer, basically, the $5 \mathrm{~W}$ questions: who was involved; what was the issue; when and where did the event occur; why does it come to mind as having been particularly effective and/or ineffective? Further, the respondent determines whether or not the event is representative of normal communication within the organization.

5. Network Analysis

One purpose of the network analysis is to ascertain where, when, how, from whom, and to whom information flows in the organization. Another purpose is to determine whether information is distorted in the flow. The auditor contrasts the communications network with the organizational chart. Often valuable disparities are exposed. Senior managers' perceptions both of their accessibility for upward communication and of the quantity and quality of information they disseminate often contrast with staff's narrations of actual experiences.

6. Content Analysis

Since a well constructed Communication Audit contains openended questions in interviews, focus groups, and questionnaires, the responses to these questions must be analysed. After reading and reviewing all responses, the auditor designates categories of responses, codes all responses, and, in the audit report, discusses each category, making observations and suggestions. In a major audit, three or more readers each categorize all responses, and the auditor calculates statistically the reliability of the coding. Detailed and insightful analysis of content will identify categories of satisfactory and unsatisfactory communication in the organization or unit under scrutiny. 


\section{Communication Diaries}

This valuable system of collecting data is costly in terms of time and attention to detail. All participants in the audit note every message they either receive or send. Further, they identify the messages according to pre-determined characteristics. If all members of the organization participate fully, the data are rich; however, seldom do all participants engage fully. If the auditor elects to use communication diaries, she will need to ensure commitment to the process from all participants prior to the audit. If all members of the organization are determined to produce a statistically valid and reliable, and organizationally useful, Communication Audit, they are likely to participate as fully as possible in communication diaries. If up-front enthusiastic commitment is not universally evident, then full participation in the diaries is not likely and they should not be used.

Only very rarely would all seven of these techniques be used in a Communication Audit. How many and which techniques comprise the audit depends on the size and culture of the unit under scrutiny and the scope of the audit as commissioned.

\section{CONCLUSION}

Organizations combine cultures, values, relationships policies, procedures, and generally accepted systems of functioning, communicating and responding to change. Individuals within organizations experience the consequences of changes to organizational functioning. The response of individuals, groups, divisions, departments, and work units to change is often determined by the degree of their participation in decisions to make changes and the manner in which they are informed of upcoming changes.

The Communication Audit is a useful procedure for determining the efficacy and efficiency of communication in an organization. Through 
the audit, the organization can identify and quantify the most salient, informative, valued, and preferred sources and channels of communication. It can then capitalize on the findings and enhance the communication process.

While teachers of technical communication cannot personally conduct Communication Audits of workplaces where our graduates function, we can be familiar with the issues and general topics of the audit, and make efforts to contextualize the communication forms we teach. By identifying an environment of discourse, we can move beyond the communication modes often isolated in, and sometimes between, courses. For example, some institutions teach in one course "The Letter and Memo," and in another "The Report." Yet another course contains "The Oral Report." Such separation fragments the environment of discourse. The concept of the Communication Audit to trace the flow of information through an organization is a valuable point of reference in Technical and Organizational Communication, as taught and practiced.

\section{REFERENCES}

Downs, Cal W. (1988). Communication Audits. Glenview, Illinois: Scott Foresman and Company.

Hopelain, D.G. (1982). "Assessing the Climate for Change: A Method of Managing Change in a System Implantation" Systems, Objectives and Solutions, Vol. 2.

Hopkins, Kevin R., Susan L. Nestleroth, and Clint Bolick (1991). Help Wanted: How Companies Can Survive and Thrive in the Coming Worker Shortage. New York: McGraw-Hill, Inc.

Kanter, Rosabeth Moss (1989). When Giants Learn to Dance: Mastering the Challenge of Strategy, Management, and Careers in the 1990's. New York: Simon and Schuster.

Leonard-Barton, Dorothy (Nov 1985). "Implementing New Technology" Harvard Business Review, Vol. 63, Nov-Dec. 
(1988). "Managerial Influence in the Implementation of New Technology" Management Science, Vol. 34, No. 10 (Oct).

MacKay, Harvey (1988). Swim with the sharks without Being Eaten Alive: Outsell, Outmanage, Outmotivate, and Outnegotiate Your Competition. New York: William Morrow and Company.

Manzies, Heather (1989). Fastforward and Out of Control: How Technology Is Changing Your Life. Toronto: Macmillan.

Naisbitt, John and Patricia Aburdene (1990). Megatrends 2000: Ten New Directions for the 1990's. New York: William Morrow and Company.

Toffler, Alvin (1990). Powershift: Knowledge, Wealth and Violence at the Edge of the 21st Century. New York: Bantam Books.

Wurman, Richard Saul (1989). Information Anxiety. New York: Doubleday.

Zuboff, Shoshana (1988). In the Age of the Smart Machine: The Future of Work and Power. New York: Basic Books, Inc. 